\title{
On Similarity and Entropy of Neutrosophic Sets
}

\author{
Pinaki Majumdar ${ }^{1,2}$ \& S.K. Samanta ${ }^{2}$ \\ ${ }^{1}$ Department of Mathematics, M.U.C Women's College, Burdwan (W.B.), India \\ ${ }^{2}$ Department of Mathematics, Visva-Bharati, Santiniketan (W.B.), India
}

\begin{abstract}
In this paper we have introduced the notion of distance between two single valued neutrosophic sets and studied its properties. We have also defined several similarity measures between them and investigated their characteristics. A measure of entropy of a single valued neutrosophic set has also been introduced.
\end{abstract}

Keywords: Single valued neutrosophic set; Hausdorff distance; similarity measure; weights; entropy; cardinality.

AMS Subject classification: 03B99, 03E99

\section{Introduction}

In many practical situations and in many complex systems like biological, behavioral and chemical etc., we encounter different types of uncertainties. Our classical mathematics does not practice any kind of uncertainty in its tools, excluding possibly the case of probability, where it can handle a particular king of uncertainty called randomness. Therefore new techniques and modification of classical tools are required to model such uncertain phenomenon. In 1965, L. A. Zadeh [14] coined his remarkable theory of Fuzzy sets that deals with a kind of uncertainty known as "Fuzziness" and which is due to partial membership of an element in a set. Later this "Fuzziness" concept leads to the highly acclaimed theory of Fuzzy Logic. After the invention of fuzzy sets many other hybrid concepts begun to develop. In 1983, K. Atanasov [1] introduced the idea of Intuitionistic fuzzy sets, a set with each member having a degree of belongingness as well as a degree of non-belongingness. This is again a generalization of fuzzy set theory. Although Fuzzy set theory is very successful in handling uncertainties arising from vagueness or partial belongingness of an element in a set, it cannot model all sorts of uncertainties prevailing in different real physical problems such as problems involving incomplete information. Hence further generalizations of fuzzy and intuitionistic fuzzy sets are required. After that many theories have been evolved which are successful in their respective domains.

Recently a new theory has been introduced and which is known as neutrosophic logic and sets. The term "neutro-sophy" means "knowledge of neutral thought" and this 'neutral' represents the main distinction between 'fuzzy' and 'intuitionistic fuzzy' logic and set. 'Neutrosophic logic' was introduced by Florentin Smarandache [10] in 1995. It is a logic in which each proposition is estimated to have a degree of truth (T), a degree of indeterminacy (I) and a degree of falsity (F). A Neutrosophic set is a set where

\footnotetext{
${ }^{1}$ Corresponding author, E-Mail:pmajumdar2@rediffmail.com
} 
each element of the universe has a degree of truth, indeterminacy and falsity respectively and which lies between $]^{-} 0,1^{+}[$, the non-standard unit interval. Unlike in intuitionistic fuzzy sets, where the incorporated uncertainty is dependent of the degree of belongingness and degree of non belongingness, here the uncertainty present, i.e. indeterminacy factor, is independent of truth and falsity values. In 2005, Wang et. al. [12] introduced an instance of neutrosophic set known as single valued neutrosophic sets (SVNS) which were motivated from the practical point of view and that can be used in real scientific and engineering applications. The single valued neutrosophic set is a generalization of classical set, fuzzy set, intuitionistic fuzzy set and paraconsistent sets etc. Again fuzziness is a feature of imperfect information which is due to partial belongingness of an element to a set. The term 'entropy' as a measure of fuzziness was first coined by Zadeh [15] in 1965. The entropy measure of fuzzy sets has many applications in areas like image processing, optimizations etc. [4, 9]. Actually a measure of imperfectness of information represented by a set is measured in terms of entropy. So measuring of entropy of single valued neutrosophic sets will be useful in cases whenever modeling of uncertain situations is done through SVNSs. On the other hand similarity is a key concept in a number of fields such as linguistics, psychology and computational intelligence. In several problems we often need to compare two sets. We are often interested to know whether two patterns or images are identical or approximately identical or at least to what degree they are identical. Similar elements are regarded from different points of view by using resemblances, distances, closeness, proximity, dissimilarities etc. So it is natural and very useful to address the issues similarity measure for this new set, viz. single valued neutrosophic set.

The rest of the paper is constructed as follows: Some preliminary definitions and results were given in section 2. In section 3, different types of distances between two single valued neutrosophic sets have been introduced. Different measures of similarity and their properties have been discussed in section 4 . In section 5 , the notion of entropy of a single valued neutrosophic set has been given. Section 6 briefly compares the methods described here with earlier available methods. Section 7 concludes the paper.

\section{Preliminaries}

In this section we recall some definitions, operations and properties regarding single valued neutrosophic sets (SVNS in short) from [12], which will be used in the rest of the paper.

A single valued neutrosophic set has been defined in [12] as follows:

Definition 2.1 Let $X$ be a universal set. A Neutrosophic set $A$ in $X$ is characterized by a truthmembership function $t_{A}$, a indeterminacy-membership function $i_{A}$ and a falsity-membership function $f_{A}$, where $t_{A}, i_{A}, f_{A}: X \rightarrow[0,1]$, are functions and $\forall x \in X, x \equiv x\left(t_{A}(x), i_{A}(x), f_{A}(x)\right) \in A$, is a single valued neutrosophic element of $A$.

A single valued neutrosophic set $A$ over a finite universe $X=\left\{x_{1}, x_{2}, x_{3}, \ldots, x_{n}\right\}$ is represented as 
$A=\sum_{i=1}^{n} \frac{x_{i}}{<t_{A}\left(x_{i}\right), i_{A}\left(x_{i}\right), f_{A}\left(x_{i}\right)>}$

In case of SVNS the degree of truth membership (T), indeterminacy membership (I) and the falsity membership(F) values lies in $[0,1]$ instead of the non standard unit interval $]^{-} 0,1^{+}[$as in the case of ordinary neutrosophic sets.

An example of SVNS is given below:

Example 2.2 Assume that $X=\left\{x_{1}, x_{2}, x_{3}\right\}$, where $x_{1}$ is capacity, $x_{2}$ is trustworthiness and, $x_{3}$ is price of a machine, be the universal set. The values of $x_{1}, x_{2}, x_{3}$ are in $[0,1]$. They are obtained from the questionnaire of some domain experts, their option could be a degree of "good service", a degree of indeterminacy and a degree of "poor service". $A$ is a single valued Neutrosophic set of $X$ defined by

$$
A=\left\langle 0.3,0.4,0.5>/ x_{1}+<0.5,0.2,0.3>/ x_{2}+<0.7,0.2,0.2>/ x_{3} .\right.
$$

Next we state the definitions of complement and containment as follows:

Definition 2.3 The complement of a SVNS $A$ is denoted by $A^{c}$ and is defined by $t_{A^{c}}(x)=f_{A}(x) ; i_{A^{c}}(x)=1-i_{A}(x) \& f_{A^{c}}(x)=t_{A}(x) \forall x \in X$.

Definition 2.4 A SVNS $A$ is contained in the other SVNS $B$, denoted as $A \subset B$, if and only if $t_{A}(x) \leq t_{B}(x) ; i_{A}(x) \leq i_{B}(x) \& f_{A}(x) \geq f_{B}(x) \forall x \in X$.

Two sets will be equal, i.e. $A=B$, iff $A \subset B \& B \subset A$.

Let us denote the collection of all SVNS in $X$ as $N(X)$.

Several operations like union and intersection has been defined on SVNS's and they satisfy most of the common algebraic properties of ordinary sets.

Definition 2.5 The union of two SVNS $A \& B$ is a SVNS $C$, written as $C=A \cup B$, which is defined as follows: $t_{C}(x)=\max \left(t_{A}(x), t_{B}(x)\right) ; i_{C}(x)=\max \left(i_{A}(x), i_{B}(x)\right) \& f_{C}(x)=\min \left(f_{A}(x), f_{B}(x)\right) \forall x \in X$.

Definition 2.6 The intersection of two SVNS $A \& B$ is a SVNS $C$, written as $C=A \cap B$, which is defined as follows: $t_{C}(x)=\min \left(t_{A}(x), t_{B}(x)\right) ; i_{C}(x)=\min \left(i_{A}(x), i_{B}(x)\right) \& f_{C}(x)=\max \left(f_{A}(x), f_{B}(x)\right) \forall x \in X$.

For practical purpose, throughout the rest of the paper, we have considered only SVNS in a finite universe. 


\section{Distance between two neutrosophic sets.}

In this section we introduce the notion of distance between two single valued neutrosophic sets $A$ and $B$ in the universe $X=\left\{x_{1}, x_{2}, x_{3}, \ldots, x_{n}\right\}$.

Definition 3.1 Let $A=\sum_{i=1}^{n} \frac{x_{i}}{<t_{A}\left(x_{i}\right), i_{A}\left(x_{i}\right), f_{A}\left(x_{i}\right)>}$ and $B=\sum_{i=1}^{n} \frac{x_{i}}{\left.<t_{B}\left(x_{i}\right), i_{B}\left(x_{i}\right), f_{B}\left(x_{i}\right)\right\rangle}$ be two single valued neutrosophic sets in $X=\left\{x_{1}, x_{2}, x_{3}, \ldots, x_{n}\right\}$. Then

- The Hamming distance between $A$ and $B$ is defined as follows:

$$
d_{N}(A, B)=\sum_{i=1}^{n}\left\{\left|t_{A}\left(x_{i}\right)-t_{B}\left(x_{i}\right)\right|+\left|i_{A}\left(x_{i}\right)-i_{B}\left(x_{i}\right)\right|+\left|f_{A}\left(x_{i}\right)-f_{B}\left(x_{i}\right)\right|\right\}
$$

- The normalized Hamming distance between $A$ and $B$ is defined as follows:

$$
l_{N}(A, B)=\frac{1}{3 n} \sum_{i=1}^{n}\left\{\left|t_{A}\left(x_{i}\right)-t_{B}\left(x_{i}\right)\right|+\left|i_{A}\left(x_{i}\right)-i_{B}\left(x_{i}\right)\right|+\left|f_{A}\left(x_{i}\right)-f_{B}\left(x_{i}\right)\right|\right\}
$$

- The Euclidian distance between $A$ and $B$ is defined as follows:

$e_{N}(A, B)=\sqrt{\sum_{i=1}^{n}\left\{\left(t_{A}\left(x_{i}\right)-t_{B}\left(x_{i}\right)\right)^{2}+\left(i_{A}\left(x_{i}\right)-i_{B}\left(x_{i}\right)\right)^{2}+\left(f_{A}\left(x_{i}\right)-f_{B}\left(x_{i}\right)\right)^{2}\right.}$

- The normalized Euclidian distance between $A$ and $B$ is defined as follows:

$q_{N}(A, B)=\sqrt{\frac{1}{3 n} \sum_{i=1}^{n}\left\{\left(t_{A}\left(x_{i}\right)-t_{B}\left(x_{i}\right)\right)^{2}+\left(i_{A}\left(x_{i}\right)-i_{B}\left(x_{i}\right)\right)^{2}+\left(f_{A}\left(x_{i}\right)-f_{B}\left(x_{i}\right)\right)^{2}\right.}$

Now for equations (1) - (4) the following holds:

(i) $0 \leq d_{N}(A, B) \leq 3 n$

(ii) $0 \leq l_{N}(A, B) \leq 1$

(iii) $0 \leq e_{N}(A, B) \leq \sqrt{3 n}$

(iv) $0 \leq q_{N}(A, B) \leq 1$

Example 3.2 Let $X=\{a, b, c, d\}$ be the universe and $A$ and $B$ be two single valued neutrosophic sets in $X$ defined as follows: 


$$
\begin{aligned}
& A=\left\{\frac{a}{<0.5,0.2,0.9>}, \frac{b}{<0.8,0.4,0.2>}, \frac{c}{<0.3,0.8,0.7>}, \frac{d}{<0.6,0.3,0.5>}\right\} . \\
& B=\left\{\frac{a}{<0.7,0.4,0.2>}, \frac{b}{<0.5,0.5,0.3>}, \frac{c}{<0.1,0.2,0.3>}, \frac{d}{<0.8,0.1,0.6>}\right\} .
\end{aligned}
$$

Then the distance between $A, B$ will be as follows:

$d_{N}(A, B)=3.3$

Similarly the other three distances will be $l_{N}(A, B)=\frac{3.3}{12}=0.275, e_{N}(A, B) \cong 1.15, q_{N}(A, B) \cong 0.33$.

Then the following result can be easily proved.

Proposition 3.3 The distances $d_{N}, l_{N}, e_{N}, q_{N}$ defined above are metric.

Definition 3.4 (Cardinality) The minimum (or sure) cardinality of a SVNS $A$ is denoted as $\min \sum \operatorname{count}(A)$ or $c^{l}$ and is defined as $c^{l}=\sum_{i=1}^{n} t_{A}\left(x_{i}\right)$. The maximum cardinality of $A$ is denoted by $\max \sum \operatorname{count}(A)$ or $c^{u}$ and is defined as $c^{u}=\sum_{i=1}^{n}\left\{t_{A}\left(x_{i}\right)+\left(1-i_{A}\left(x_{i}\right)\right)\right\}$. The cardinality of $A$ is defined by the interval $\left[c^{l}, c^{u}\right]$.

Example 3.5 For the SVNS $A$ given in example 3.2 we have the following:

$$
\begin{aligned}
& c^{l}=\sum_{i=1}^{n} t_{A}\left(x_{i}\right)=0.5+0.8+0.3+0.6=2.2 \quad \& \\
& c^{u}=\sum_{i=1}^{n}\left\{t_{A}\left(x_{i}\right)+\left(1-i_{A}\left(x_{i}\right)\right)\right\}=1.3+1.4+0.5+1.3=4.5 .
\end{aligned}
$$

\section{Similarity Measure between two single valued neutrosophic sets}

In this section we define the notion of similarity between two SVNSs. We have adopted various methods for calculating this similarity. The first method is based on distances defined in the previous section. The second one is based on a matching function and the last one is based on membership grades. 
In general a similarity measure between two SVNSs is a function defined as $s: N(X)^{2} \rightarrow[0,1]$ which satisfies the following properties:

(i) $S(A, B) \in[0,1]$,

(ii) $S(A, B)=1 \Leftrightarrow A=B$,

(iii) $S(A, B)=S(B, A)$,

(iv) $A \subset B \subset C \Rightarrow S(A, C) \leq S(A, B) \wedge S(B, C)$

But individual measures may satisfy more properties in addition to (9).

Now similarity can be calculated using several techniques. Here we have adopted three common techniques namely, the distance based, the one based on a matching function and lastly on membership grade based.

\subsection{Distance based similarity measure}

We know that similarity is inversely proportional with the distance between them. Using the distances defined in equation (1) - (4) we define measures of similarity $s^{1}$ between two SVN sets $A$ and $B$ as follows:

$s^{1}(A, B)=\frac{1}{1+d(A, B)}$

For example if we use Hamming distance $d_{N}$ then the associated measure of similarity will be denoted by $s_{N}^{1}$ and is defined by:

$s_{N}^{1}(A, B)=\frac{1}{1+d_{N}(A, B)}$

The following example calculates the similarity measure between the two SVNSs:

Example 4.1.1 The similarity measure between the two SVNSs defined in example 3.2 will be

$s^{1}(A, B)=\frac{1}{1+3.3} \cong 0.233$

Proposition 4.1.2 The distance based similarity measure $s^{1}$, between two SVNSs $A$ and $B$ satisfies the following properties: 
(i) $0 \leq s^{1}(A, B) \leq 1$

(ii) $s^{1}(A, B)=1$ iff $A=B$

(iii) $s^{1}(A, B)=s^{1}(B, A)$

(iv) $A \subset B \subset C \Rightarrow S(A, C) \leq S(A, B) \wedge S(B, C)$

Proof. The results (i)- (iii) holds trivially from definition. We only prove (iv).

Let $A \subset B \subset C$. Then we have

$t_{A}(x) \leq t_{B}(x) \leq t_{c}(x) ; i_{A}(x) \leq i_{B}(x) \leq i_{c}(x) \& f_{A}(x) \geq f_{B}(x) \geq f_{c}(x) \forall x \in U$. Now

$\left|t_{A}(x)-t_{B}(x)\right| \leq\left|t_{A}(x)-t_{c}(x)\right|$ and $\left|t_{B}(x)-t_{C}(x)\right| \leq\left|t_{A}(x)-t_{C}(x)\right|$ will hold.

Similarly, $\left|i_{A}(x)-i_{B}(x)\right| \leq\left|i_{A}(x)-i_{c}(x)\right| \&\left|i_{B}(x)-i_{C}(x)\right| \leq\left|i_{A}(x)-i_{C}(x)\right|$ and

$\left|f_{A}(x)-f_{B}(x)\right| \leq\left|f_{A}(x)-f_{c}(x)\right| \&\left|f_{B}(x)-f_{C}(x)\right| \leq\left|f_{A}(x)-f_{C}(x)\right|$ holds.

Thus

$d(A, B) \leq d(A, C) \Rightarrow s^{1}(A, B) \geq s^{1}(A, C)$ and $d(B, C) \leq d(A, C) \Rightarrow s^{1}(B, C) \geq s^{1}(A, C)$

$\Rightarrow s^{1}(A, C) \leq s^{1}(A, B) \wedge s^{1}(B, C)$.

This is true for all the distance functions defined in equations (1) to (4).

Hence the result.

\subsection{Similarity measure based on membership degrees}

Another measure of similarity $s^{2}$ between two SVN sets $A$ and $B$ could be defined as follows:

$$
s^{2}(A, B)=\frac{\sum_{i=1}^{n}\left\{\min \left\{t_{A}\left(x_{i}\right), t_{B}\left(x_{i}\right)\right\}+\min \left\{i_{A}\left(x_{i}\right), i_{B}\left(x_{i}\right)\right\}+\min \left\{f_{A}\left(x_{i}\right), f_{B}\left(x_{i}\right)\right\}\right\}}{\sum_{i=1}^{n}\left\{\max \left\{t_{A}\left(x_{i}\right), t_{B}\left(x_{i}\right)\right\}+\max \left\{i_{A}\left(x_{i}\right), i_{B}\left(x_{i}\right)\right\}+\max \left\{f_{A}\left(x_{i}\right), f_{B}\left(x_{i}\right)\right\}\right.}
$$

Example 4.2.1 Here the similarity measure between the two SVN sets defined in example 3.2 will be

$$
s^{2}(A, B)=\frac{3.8}{7.1} \cong 0.535
$$


Proposition 4.2.2 The distance based similarity measure $s^{2}$, between two SVNS $A$ and $B$ satisfies the following properties:

(i) $0 \leq s^{2}(A, B) \leq 1$

(ii) $s^{2}(A, B)=1$ iff $A=B$

(iii) $s^{2}(A, B)=s^{2}(B, A)$

(iv) $A \subset B \subset C \Rightarrow s^{3}(A, C) \leq s^{3}(A, B) \wedge s^{3}(B, C)$.

Proof. Properties (i) and (iii) follows readily from definition.

(ii) It is clear that if $A=B \Rightarrow s^{2}(A, B)=1$.

Conversely, let

$s^{2}(A, B)=1 \Rightarrow \frac{\sum_{i=1}^{n}\left\{\min \left\{t_{A}\left(x_{i}\right), t_{B}\left(x_{i}\right)\right\}+\min \left\{i_{A}\left(x_{i}\right), i_{B}\left(x_{i}\right)\right\}+\min \left\{f_{A}\left(x_{i}\right), f_{B}\left(x_{i}\right)\right\}\right\}}{\sum_{i=1}^{n}\left\{\max \left\{t_{A}\left(x_{i}\right), t_{B}\left(x_{i}\right)\right\}+\max \left\{i_{A}\left(x_{i}\right), i_{B}\left(x_{i}\right)\right\}+\max \left\{f_{A}\left(x_{i}\right), f_{B}\left(x_{i}\right)\right\}\right.}=1$

$\Rightarrow \sum_{x}\left\{\min \left\{t_{A}\left(x_{i}\right), t_{B}\left(x_{i}\right)\right\}+\min \left\{i_{A}\left(x_{i}\right), i_{B}\left(x_{i}\right)\right\}+\min \left\{f_{A}\left(x_{i}\right), f_{B}\left(x_{i}\right)\right\}\right\}$

$=\sum_{x}\left\{\max \left\{t_{A}\left(x_{i}\right), t_{B}\left(x_{i}\right)\right\}+\max \left\{i_{A}\left(x_{i}\right), i_{B}\left(x_{i}\right)\right\}+\max \left\{f_{A}\left(x_{i}\right), f_{B}\left(x_{i}\right)\right\}\right.$

$\Rightarrow \sum_{x}\left\{\left[\min \left\{t_{A}\left(x_{i}\right), t_{B}\left(x_{i}\right)\right\}-\max \left\{t_{A}\left(x_{i}\right), t_{B}\left(x_{i}\right)\right\}\right]+\left[\min \left\{i_{A}\left(x_{i}\right), i_{B}\left(x_{i}\right)\right\}-\max \left\{i_{A}\left(x_{i}\right), i_{B}\left(x_{i}\right)\right\}\right]+\right.$ $\min \left[\left\{f_{A}\left(x_{i}\right), f_{B}\left(x_{i}\right)\right\}-\max \left\{f_{A}\left(x_{i}\right), f_{B}\left(x_{i}\right)\right\}\right]=0$

Thus for each $x$,

$\min \left\{t_{A}\left(x_{i}\right), t_{B}\left(x_{i}\right)\right\}-\max \left\{t_{A}\left(x_{i}\right), t_{B}\left(x_{i}\right)\right\}=0, \min \left\{i_{A}\left(x_{i}\right), i_{B}\left(x_{i}\right)\right\}-\max \left\{i_{A}\left(x_{i}\right), i_{B}\left(x_{i}\right)\right\}=0 \&$ $\min \left\{f_{A}\left(x_{i}\right), f_{B}\left(x_{i}\right)\right\}-\max \left\{f_{A}\left(x_{i}\right), f_{B}\left(x_{i}\right)\right\}=0$ holds.

Thus $t_{A}(x)=t_{B}(x), i_{A}(x)=i_{B}(x) \& f_{A}(x)=f_{B}(x) \Rightarrow A=B$.

(iv) Now we prove the last result.

Let $A \subset B \subset C$. Then we have

$t_{A}(x) \leq t_{B}(x) \leq t_{c}(x) ; i_{A}(x) \leq i_{B}(x) \leq i_{c}(x) \& f_{A}(x) \geq f_{B}(x) \geq f_{c}(x) \forall x \in U$. Now 


$$
\begin{aligned}
& t_{A}(x)+i_{A}(x)+f_{B}(x) \geq t_{A}(x)+i_{A}(x)+f_{C}(x) \text { and } \\
& t_{B}(x)+i_{B}(x)+f_{A}(x) \leq t_{C}(x)+i_{C}(x)+f_{A}(x) \\
& \therefore s^{2}(A, B)=\frac{t_{A}(x)+i_{A}(x)+f_{B}(x)}{t_{B}(x)+i_{B}(x)+f_{A}(x)} \geq \frac{t_{A}(x)+i_{A}(x)+f_{C}(x)}{t_{C}(x)+i_{C}(x)+f_{A}(x)}=s^{2}(A, C)
\end{aligned}
$$

Again similarly we have:

$$
\begin{aligned}
& t_{B}(x)+i_{B}(x)+f_{C}(x) \geq t_{A}(x)+i_{A}(x)+f_{C}(x) \& \\
& t_{C}(x)+i_{C}(x)+f_{A}(x) \geq t_{C}(x)+i_{C}(x)+f_{B}(x) \\
& \therefore s^{2}(B, C)=\frac{t_{B}(x)+i_{B}(x)+f_{C}(x)}{t_{C}(x)+i_{C}(x)+f_{B}(x)} \geq \frac{t_{A}(x)+i_{A}(x)+f_{C}(x)}{t_{C}(x)+i_{C}(x)+f_{A}(x)}=s^{2}(A, C) \\
& \Rightarrow s^{2}(A, C) \leq s^{2}(A, B) \wedge s^{2}(B, C) .
\end{aligned}
$$

Hence the proof of this proposition is complete.

\subsection{Similarity measure based on a matching function}

Next consider a universe where each element $x_{i}$ has a weight $\omega_{i}$. Then we require a new measure of similarity different from those discussed earlier.

Often weights are associated with each element of an universe to give an order of importance among the elements. To illustrate the situation we give an example:

Suppose that there is a system to detect a disease based on several symptoms associated with it. Now each symptoms is characterized by three things namely a degree of truth, a degree of indeterminacy and a degree of falsity. So for each disease we can have a corresponding single valued neutrosophic set with symptoms as its elements. These SVNSs will act as our knowledgebase. Whenever a patient comes with some health problem the system will generate his corresponding SVNS. The measure of similarity, as discussed in previous sections, between these SVNSs can detect the possible disease. But we know that many diseases have several symptoms in common. So for a specific disease all the symptoms are not equally important. Here we can assign weights to each symptom corresponding to a particular disease. So we have a universe of symptoms where each symptom has its corresponding weights. In this case we cannot use similarity measures described in 4.1 and 4.2 .

Again a suitable function is often used to measure the similarity between two sets which is called a matching function. Chen [2,3] first introduced the notion of matching function. Here a weighted similarity measure $S^{w}$ between $A$ and $B$ has been defined using a matching function as follows:

$$
s^{w}(A, B)=\frac{\sum_{i=1}^{n} \omega_{i}\left(t_{A}\left(x_{i}\right) \cdot t_{B}\left(x_{i}\right)+i_{A}\left(x_{i}\right) \cdot i_{B}\left(x_{i}\right)+f_{A}\left(x_{i}\right) \cdot f_{B}\left(x_{i}\right)\right)^{2}}{\sum_{i=1}^{n} \omega_{i}\left\{\left(t_{A}\left(x_{i}\right)^{2}+i_{A}\left(x_{i}\right)^{2}+f_{A}\left(x_{i}\right)^{2}\right) \cdot\left(t_{B}\left(x_{i}\right)^{2}+i_{B}\left(x_{i}\right)^{2}+f_{B}\left(x_{i}\right)^{2}\right)\right\}} . .
$$


Example 4.3.1 Consider the two SVN sets in example 3.2. Further let the elements $a, b, c, d$ of the universe $\mathrm{X}$ have weights $0.1,0.3,0.5,0.2$ respectively.

Then the weighted similarity measure between the two SVN sets will be

$s^{w}(A, B)=\frac{0.1 \times 0.3721+0.3 \times 0.4356+0.5 \times 0.16+0.2 \times 0.6561}{0.1 \times 0.759+0.3 \times 0.4956+0.5 \times 0.1708+0.2 \times 0.707}=\frac{0.37911}{0.45138} \cong 0.84$

Proposition 4.3.2 The weighted similarity measure $s^{w}$, between two SVNS $A$ and $B$ satisfies the following properties:

(i) $0 \leq s^{w}(A, B) \leq 1$

(ii) $s^{w}(A, B)=1$ if $A=B$

(iii) $s^{w}(A, B)=s^{w}(B, A)$

Proof. Trivially follows from definition and Cauchy-Schawarz inequality.

Note that here the result (ii) and (iv) (i.e. monotonacity law) of equation (9) will not hold due to the effects of the weights.

\section{Entropy of a Single Valued Neutrosophic Set}

Entropy can be considered as a measure of uncertainty involved in a set, whether fuzzy or intuitionistic fuzzy or vague etc. Here the SVNS are also capable of handling uncertain data, therefore as a natural consequence we are also interested in finding the entropy of a single valued neutrosophic set.

Entropy as a measure of fuzziness was first mentioned by Zadeh [15] in 1965. Later De Luca-Termini [5] axiomatized the non-probabilistic entropy. According to them the entropy $E$ of a fuzzy set A should satisfy the following axioms:

(DT1) $E(A)=0$ iff $A \in 2^{X}$

$(D T 2) E(A)=1$ iff $\mu_{A}(x)=0.5, \forall x \in X$

$(D T 3) E(A) \leq E(B)$ iff Ais less fuzzy than $B$, i.e. if $\mu_{A}(x) \leq \mu_{B}(x) \leq 0.5 \forall x \in X$.

or if $\mu_{A}(x) \geq \mu_{B}(x) \geq 0.5, \forall x \in X$.

Seve

$(D T 4) E\left(A^{c}\right)=E(A)$.

ral

othe

$r$ authors have investigated the notion of entropy. Kaufmann [6] proposed a distance based measure of soft entropy; Yager [13] gave another view of degree of fuzziness of any fuzzy set in terms of lack of distinction between the fuzzy set and its complement. Kosko [7] investigated the fuzzy entropy in relation to a measure of subset hood. Szmidt \& Kacprzyk [11] studied the entropy of intuitionistic fuzzy sets. Majumdar and Samanta [8] investigated the entropy of soft sets etc. 
Definition 5.1 Similarly here in case of SVNS also we introduce the entropy as a function $E_{N}: N(X) \rightarrow[0,1]$ which satisfies the following axioms:

(i) $E_{N}(A)=0$ if $A$ is a crisp set

(ii) $E_{N}(A)=1$ if $\left(t_{A}(x), i_{A}(x), f_{A}(x)\right)=(0.5,0.5,0.5) \forall x \in X$

(iii) $E_{N}(A) \geq E_{N}(B)$ if $A$ more uncertain than $B$,

i.e. $t_{A}(x)+f_{A}(x) \leq t_{B}(x)+f_{B}(x)$ and $\left|i_{A}(x)-i_{A^{c}}(x)\right| \leq\left|i_{B}(x)-i_{B^{c}}(x)\right|$

(iv) $E_{N}(A)=E_{N}\left(A^{c}\right) \forall A \in N(X)$.

Now notice that in a SVNS the presence of uncertainty is due to two factors, firstly due to the partial belongingness and partial non-belongingness and secondly due to the indeterminacy factor. Considering these two factors we propose an entropy measure $E_{1}$ of a single valued neutrosophic sets $A$ as follows:

$E_{1}(A)=1-\frac{1}{n} \sum_{x_{i} \in X}\left(t_{A}\left(x_{i}\right)+f_{A}\left(x_{i}\right)\right) \cdot\left|i_{A}\left(x_{i}\right)-i_{A^{c}}\left(x_{i}\right)\right|$

Proposition $5.2 E_{1}$ satisfies all the axioms given in definition 5.1.

Proof (i) For a crisp set $A$ and $i_{A}(x)=0 \forall x \in X$. Hence $E_{1}(A)=0$ holds.

(ii) If $A$ be such that $\left(t_{A}(x), i_{A}(x), f_{A}(x)\right)=(0.5,0.5,0.5) \forall x \in X$,

then $t_{A}(x)+f_{A}(x)=1$ and $i_{A}(x)-i_{A^{c}}(x)=0.5-0.5=0 \forall x \in X \Rightarrow E_{1}(A)=1$

(iii) It holds from definition.

(iv) $E_{1}(A)=E_{1}\left(A^{c}\right)$ holds obviously from definition.

Thus $E_{1}$ is an entropy function defined on $N(X)$.

Example 5.3 Let $X=\{a, b, c, d\}$ be the universe and $A$ be a single valued neutrosophic set in $X$ defined as follows:

$$
A=\left\{\frac{a}{<0.5,0.2,0.9>}, \frac{b}{<0.8,0.4,0.2>}, \frac{c}{<0.3,0.8,0.7>}, \frac{d}{<0.6,0.3,0.5>}\right\} .
$$

Then the entropy of $A$ will be $E_{1}(A)=1-0.52=0.48$. 


\section{Discussions}

The techniques of similarity and entropy described here are totally new concepts for single valued neutrosophic sets. For the case of entropy no earlier methods of measuring entropy will be able to determine the entropy of a SVNSs, as here the sum of the degrees of truth, indeterminacy and falsity is not necessarily bounded. A fuzzy set $F$ can be considered as a SVNS with degree of indeterminacy and degree of falsity zero, then its entropy will be $E_{1}(F)=1-\frac{1}{n} \sum_{x_{i} \in X}\left(t_{A}\left(x_{i}\right)\right)$. For crisp set this value is 1 , which is conformable with earlier known result.

The similarity measurements described here are again totally new concepts for SVNSs. These measures can also be applied to measure the similarity of intuitionistic fuzzy sets (IFS) where the indeterminacy factor $i$ should be replaced by $\pi=1-t-f$ in case of IFS.

\section{Conclusion}

The recently proposed notion of neutrosophic sets is a general formal framework for studying uncertainties arising due to 'indeterminacy' factors. From the philosophical point of view, it has been shown that a neutrosophic set generalizes a classical set, fuzzy set, interval valued fuzzy set, intuitionistic fuzzy set etc. A single valued neutrosophic set is an instance of neutrosophic set which can be used in real scientific and engineering applications. Therefore the study of single valued neutrosophic sets and its properties have a considerable significance in the sense of applications as well as in understanding the fundamentals of uncertainty. In this paper we have introduced some measures of similarity and entropy of single valued neutrosophic sets for the first time. These measures are consistent with similar considerations for other sets like fuzzy sets and intuitionistic fuzzy sets etc.

Acknowledgement: The authors sincerely thankful to the anonymous reviewers for their helpful comments which has helped to rewrite the paper in its present form.

\section{References}

[1] Atanasov, K, Intuitionistic Fuzzy Sets. Fuzzy Sets \& Systems, 20 (1986) pp 87-96

[2] Chen, S.M., A new approach to handling fuzzy decision making problems, IEEE Transactions on Systems, Man and Cybernet, 18(1988)1012-1016

[3] Chen, S.M., Hsiao, P.H., A Comparison of similarity measures of fuzzy values, Fuzzy Sets and Systems, 
72(1995), 79-89

[4] Cheng, C. C., Liao, K. H., Parameter optimization based on entropy weight and triangular fuzzy number, Int. J. of Engineering and Industries, 2 (2) (2011) 62-75

[5 ] De Luca, A., Termini, S., A definition of a non-probabilistic entropy in the setting of fuzzy sets theory, Information \& Control, 20 (1972), 301-312

[6] Kaufmann, A., Introduction to the theory of Fuzzy Subsets-Vol 1: Fundamental Theoretical Elements, Academic Press, New York (1975).

[7] Kosoko, B. , Fuzzy entropy and conditioning, Information Science, 40 (2) (1986) 165-174

[8] Majumdar, P., Samanta, S., Softness of a soft set: Soft set entropy, accepted in Annals of fuzzy math. and informatics, 2012.

[9] Pasha, E., Fuzzy entropy as cost function in image processing, Proc. of the $2^{\text {nd }}$ IMT-GT regional conf. on math., Stat. and appl., Universiti Sains Malaysia, Penang, 2006.

[10] Smarandache, F. , A Unifying Field in Logics. Neutrosophy: Neutrosophic Probability, Set \& Logic. Rehoboth: American Research Press, (1999).

[11] Szmidt, E., Kacprzyk, J., Entropy for intuitionistic fuzzy sets, Fuzzy Sets \& systems, 118 (2001) 467477.

[12] Wang, H. et al., Single valued neutrosophic sets, Proc. Of $10^{\text {th }}$ Int. conf. on Fuzzy Theory and Technology, Salt Lake City, Utah, July 21-26 (2005).

[13] Yagar, R. R., On the measure of fuzziness and negation, Part I: Membership in the unit interval, International Journal of General Systems, 5(1979) 189-200.

[14] Zadeh, L., Fuzzy sets. Information and Control. 8 (1965), pp 87-96.

[15] Zadeh, L., Fuzzy sets and systems, in: Proc. Symp. on Systems Theory, Polytechnic Institute of Brooklyn, New York, 1965. 